\title{
THE GENERALIZED TWO DIMENSIONAL THERMAL-ELECTRO-ELASTIC SOLUTION FOR THE CRACKED-HALF-ELLIPTICAL-HOLE PROBLEM IN A HALF PLANE*
}

\author{
Y. J. WANG \\ College of Engineering, Nanjing Agricultural University, Nanjing 210031, China \\ State key Laboratory of Mechanics and Control of Mechanical Structures, \\ Nanjing University of Aeronautics \& Astronautics, Nanjing 210016, China

$$
\text { C. F.GaO, H. P. Song, S. C. XInG }
$$

State key Laboratory of Mechanics and Control of Mechanical Structures, Nanjing University of Aeronautics \& Astronautics, Nanjing 210016, China,

$$
\text { e-mail: cfgao@nuaa.edu.cn }
$$

[Received 01 December 2014. Accepted 15 June 2015]

\begin{abstract}
The half elliptical hole with an edge crack in a thermopiezoelectric material is studied by using the complex variable method. First, the mapping function which maps the outside of the elliptical hole and the crack in the right half plane into the outside of a circular hole in a full plane is given by the method of conformal mapping. Then, the complex potential functions and the field intensity factors (FIF) are presented according to the boundary conditions, respectively. Some useful results can be found by numerical analysis: 1) The influence of the heat flux on FIF depends on the model of the crack; 2) The shape and the size of the hole possess a significant effect on the field distribution at the crack tip.

KEY WORDS: Piezoelectric material, elliptical hole, edge crack, thermoelectroelasticity.
\end{abstract}

\section{Introduction}

As a sensor or an actuator in smart structures and devices, piezoelectric materials sometimes or always work in the environment of large temperature

\footnotetext{
${ }^{*}$ Corresponding author e-mail: cfgao@nuaa.edu.cn

The work was supported by the National Natural Science Foundation of China (Nos. 11232007 and 11202099), the Science and Technology Innovation Fund for the Youth of Nanjing Agricultural University (No. KJ2013040) and the Scientific Research Foundation, Nanjing Agricultural University (No. RCQD13-06).
} 
difference, and thus, the heat flux becomes one of the important factors, which will influence the life of the piezoelectric materials. Therefore, it's very important to study the effect of heat flux on the piezoelectric structures.

In fact, the thermo-electro-elastic coupling problems have been studied by many researchers in the past decades [1-5]. More recently, Gao et al. [6] derived the exact solution for the problem of an elliptical hole or a crack in a thermopiezoelectric solid by using the complex variable method. Hesabe et al. [7] used a rational mapping function to analyze the problems of the electric current, the Joule heat, the temperature, the heat flux and the thermal stress induced by an electric current in an infinite thin-plate, containing an elliptical hole with an edge crack. Gao and Wang [8] studied the 2D problem of thermopiezoelectric materials with cracks by means of Parton assumption.

For the cracked hole problems, Wang and Gao [9] studied the problems of mode III cracks, originating from the edge of a circular hole in a piezoelectric solid under mechanical-electric loadings, by using the improved mapping function [10]. Guo et al. obtained the exact solution for anti-plane problem of two asymmetrical edge cracks, emanating from an elliptical hole in a piezoelectric material under electrically impermeable [11] and permeable [12] assumption. Hasebe [13] analyzed the stress intensity factors of cracks, initiating from a rhombic hole under uniform heat flux. Vinh et al. [14] dealt with the interaction between a cracked hole and a line crack under uniform heat flux. Based on the complex variable method, the thermoelectroelastic solution is derived for the generalized two-dimensional problem of edge cracks originating from an elliptical hole in a piezoelectric solid by Wang and Gao [15]. However, the fracture problem in the half plane with an edge cracked hole remains unclear.

Some literature can be found in the half plane problems, such as: $\mathrm{Lu}$ [16] presented the analytical solution for the half plane under the external uniform force at the edge of the half plane by the complex variable method. Gao and Liu [17] mapped the half plane of elliptical hole into the whole half plane, and the complex function for the half plane containing a half elliptical hole under the uniform positive pressure at the edge was derived. By using the complex variable method, Hasebe et al. [18] studied the half plane, containing an oblique crack at the edge, and the analytical solution was presented. However, no literature can be found on the problems of a half plane with an edge cracked hole.

In summary, no work can be found for the study of edge cracks, originating from a half elliptical hole in the half plane of piezoelectric materials under 
the uniform heat flux. Therefore, it is the purpose of this work to investigate the generalized 2D thermoelectroelastic problem of the crack, originating from a half elliptical hole at the edge of a semi-infnite piezoelectric solid, by using complex variable method. In this paper, some basic equations are summarized after the introduction, and then the complex potential functions in the thermal field and mechanical-electric field are derived, respectively. In order to find a way to decrease the field intensity factors (FIF), some numerical analyses are presented to discuss the influences of crack length and heat flux on the field quantities and the FIF. Finally, this work is concluded.

\section{Basic equations}

The thermopiezoelastic medium subjected by heat flux, mechanical and electric loadings at infinity in a fixed rectangular coordinate system $x_{j}(j=$ $1,2,3)$ is studied. The complete set of governing equations without body force, extrinsic bulk charge and heat source for thermopiezoelastic problem can be expressed as follows:

\subsection{Basic equations for the temperature field}

The governing field equation and the constitutive equation can be expressed as:

$$
\begin{gathered}
q_{i, i}=0, \\
q_{i}=-\lambda_{i j} T_{, j},
\end{gathered}
$$

where, $i, j=1,2, q_{i}$ is the heat flux, $\lambda_{i j}$ is the thermal conductivity, and $T$ stands for the temperature. From Eqs. (1) and (2), the temperature field is obtained as [8]:

$$
T=2 \operatorname{Re}\left[g^{\prime}\left(z_{t}\right)\right] \quad\left(z_{t}=x_{1}+\mu_{t} x_{2}\right),
$$

where $\mu_{t}=\left(-\lambda_{12}+i \kappa_{t}\right) / \lambda_{22}, \kappa_{t}=\left(\lambda_{11} \lambda_{22}-\lambda_{12}^{2}\right)^{1 / 2}, \lambda_{11} \lambda_{22}-\lambda_{12}^{2}>0$.

Substituting Eq. (3) into Eq. (2) yields:

$$
q_{1}=2 \operatorname{Re}\left[i \mu_{t} \kappa_{t} g^{\prime \prime}\left(z_{t}\right)\right]
$$

$$
q_{2}=-2 \operatorname{Re}\left[i \kappa_{t} g^{\prime \prime}\left(z_{t}\right)\right]
$$


The normal heat flux can be determined by:

$$
q_{n} d s=q_{1} d x_{2}-q_{2} d x_{1}
$$

the total heat can be expressed as:

$$
Q=\int q_{n} d s .
$$

Substituting Eqs. (4-6) into Eq. (7) yields:

$$
Q=2 \operatorname{Re}\left[i \kappa_{t} g^{\prime}\left(z_{t}\right)\right]
$$

The assumption that there is no heat exchange on the boundary is taken, and the normal heat flux becomes equal to zero:

$$
q_{n}=0 .
$$

In this case, one obtains from Eqs. (7-9), that:

$$
2 \operatorname{Re}\left[i \kappa_{t} g^{\prime}\left(z_{t}\right)\right]=0 .
$$

\subsection{Basic equations for the mechanical-electric field}

The governing field equations can be given by:

$$
\begin{gathered}
\sigma_{i j}=c_{i j k l} \gamma_{k l}-e_{i j s} E_{s}-\beta_{i j} T, \\
D_{i}=\varepsilon_{i s} E_{s}+e_{i r s} \gamma_{r s}+\tau_{i} T,
\end{gathered}
$$

where $i, j, k, l, s, r=1,2,3, c_{i j k l}=c_{j i k l}=c_{i j l k}=c_{k l i j}, e_{k i j}=e_{k j i}, \varepsilon_{i j}=\varepsilon_{j i}$, $\beta_{i j}=\beta_{j i}$.

In Eqs. (11) and (12), repeated indices imply summation, and $\sigma, \gamma, \mathbf{D}$, $\mathbf{E}, T$ are the stress, the strain, the electric displacement, the electric field and the temperature, respectively. $c_{i j k l}, e_{k i j}, \varepsilon_{i j}, \beta_{i j}$ and $\tau_{i}$ stand for the elastic constant, the piezoelectric coupling coefficient, the dielectric permittivity, the stress-temperature coefficients and the pyroelectric coefficient, respectively.

It is assumed, that the crack and the hole are both electrically impermeable and mechanically free, which can be expressed as:

$$
\begin{aligned}
& \mathbf{t}_{i}=\mathbf{0} \quad(i=1,2,3) \\
& D_{n}=0
\end{aligned}
$$


where $\mathbf{t}$ is the traction along the surface of the boundary. For the cracked hole problem, Eq. (13) is not exactly satisfied in the physical plane at the point, where the crack originates, due to the limitation of mapping function (the mapping function cannot maps the unsmoothed curve exactly). However, Eq. (13) is satisfied in the mathematic plane, where the junction surface of crack and the hole has been smoothed by the mapping function.

Substituting Eqs. (11) and (12) into the balance equations:

$$
\sigma_{i j, j}=0,
$$

$$
D_{i, i}=0
$$

yields:

$$
\begin{aligned}
& \left(c_{i j r s} u_{r}+e_{s j i} \phi\right)_{, s i}-\beta_{i j} T_{, i}=0, \\
& \left(-\varepsilon_{i s} \phi+e_{i r s} u_{r}\right)_{, s i}+\tau_{i} T_{, i}=0,
\end{aligned}
$$

where: $\gamma_{i j}=\left(u_{i, j}+u_{j, i}\right) / 2$ and $E_{i}=-\phi_{, i}$ have been used. $u_{i}$ stands for the displacement, and $\phi$ stands for the electric potential.

We introduce the generalized displacement function $\mathbf{u}=\left(u_{1}, u_{2}, u_{3}, \phi\right)^{T}$ and generalized stress function $\varphi=\left(\varphi_{1}, \varphi_{2}, \varphi_{3}, \varphi_{4}\right)^{T}$. The solution to Eqs. (16)and (17) can be expressed as the form of a particular solution plus a general solution.

The general solution to Eqs. (16) and (17) can be expressed as [19]:

$$
\begin{aligned}
& \mathbf{u}_{h}=\mathbf{A f}\left(z_{*}\right)+\overline{\mathbf{A}} \overline{\mathbf{f}\left(z_{*}\right)}, \\
& \boldsymbol{\varphi}_{h}=\mathbf{B f}\left(z_{*}\right)+\overline{\mathbf{B}} \overline{\mathbf{f}\left(z_{*}\right)},
\end{aligned}
$$

where: $\mathbf{f}\left(z_{*}\right)=\left[f_{1}\left(z_{1}\right), f_{2}\left(z_{2}\right), f_{3}\left(z_{3}\right), f_{4}\left(z_{4}\right)\right]^{T}, z_{i}=x_{1}+\mu_{i} x_{2}(i=1,2,3,4)$. A and $\mathbf{B}$ are two $4 \times 4$ matrices, $f_{i}\left(z_{i}\right)$ are the complex potentials to be found, $\mu_{i}$ are the complex eigenvalues with positive imaginary parts and can be obtained from the equation:

$$
\left|\mathbf{D}_{*}(\mu)\right|=0,
$$


where:

$$
\begin{gathered}
\mathbf{D}_{*}(\mu)=\mathbf{S}+\mu\left(\mathbf{R}+\mathbf{R}^{T}\right)+\mu^{2} \mathbf{W} \\
\mathbf{S}=\left[\begin{array}{cc}
\mathbf{S}_{0} & \mathbf{e}_{11} \\
\mathbf{e}_{11}^{T} & -\varepsilon_{11}
\end{array}\right], \mathbf{R}=\left[\begin{array}{cc}
\mathbf{R}_{0} & \mathbf{e}_{21} \\
\mathbf{e}_{12}^{T} & -\varepsilon_{12}
\end{array}\right], \mathbf{W}=\left[\begin{array}{cc}
\mathbf{W}_{0} & \mathbf{e}_{22} \\
\mathbf{e}_{22}^{T} & -\varepsilon_{22}
\end{array}\right],
\end{gathered}
$$

and $\left(\mathbf{S}_{0}\right)_{i k}=c_{i 1 k 1},\left(\mathbf{R}_{0}\right)_{i k}=c_{i 1 k 2},\left(\mathbf{W}_{0}\right)_{i k}=c_{i 2 k 2},(i, k=1,2,3), \mathbf{e}_{i k}=$ $\left(e_{i 1 k} e_{i 2 k} e_{i 3 k}\right)^{T},(i, k=1,2)$.

Matrixes $\mathbf{A}$ and $\mathbf{B}$ are nonsingular and they have the following relation [19], if $\mu_{i}$ are distinct:

$$
\left[\begin{array}{ll}
\mathbf{B}^{T} & \mathbf{A}^{T} \\
\overline{\mathbf{B}}^{T} & \overline{\mathbf{A}}^{T}
\end{array}\right]\left[\begin{array}{ll}
\mathbf{A} & \overline{\mathbf{A}} \\
\mathbf{B} & \overline{\mathbf{B}}
\end{array}\right]=\left[\begin{array}{ll}
\mathbf{I} & \mathbf{0} \\
\mathbf{0} & \mathbf{I}
\end{array}\right] .
$$

The particular solution can be expressed as [20]:

$$
\begin{aligned}
& \mathbf{u}_{p}=2 \operatorname{Re}\left[\mathbf{c} g\left(z_{t}\right)\right], \\
& \boldsymbol{\varphi}_{p}=2 \operatorname{Re}\left[\mathbf{d} g\left(z_{t}\right)\right],
\end{aligned}
$$

where $\mathbf{c}$ and $\mathbf{d}$ are two heat eigenvectors, which can be determined by the following equations:

$$
\begin{gathered}
\mathbf{D}_{*}\left(\mu_{t}\right) \mathbf{c}=\beta_{1}+\mu_{t} \beta_{2}, \\
\mathbf{d}=\left(\mathbf{R}^{T}+\mu_{t} \mathbf{W}\right) \mathbf{c}-\beta_{2}, \\
\beta_{1}=\left(\beta_{11}, \beta_{21}, \beta_{31}, \tau_{1}\right)^{T}, \\
\beta_{2}=\left(\beta_{12}, \beta_{22}, \beta_{32}, \tau_{2}\right)^{T} .
\end{gathered}
$$

Then, the solution to Eqs. (16) and (17) can be finally expressed as [21]:

$$
\begin{aligned}
& \mathbf{u}=2 \operatorname{Re}[\mathbf{A f}(z)+\mathbf{c} g(z)], \\
& \boldsymbol{\varphi}=2 \operatorname{Re}[\mathbf{B f}(z)+\mathbf{d} g(z)],
\end{aligned}
$$


where the condition, that for an arbitrary point on the boundary, the corresponding points $z_{t}$ and $z_{i}(i=1-4)$ can be translated into an identical point, has been assumed. In this condition, the boundary equation can be reduced to that containing one variable, and thus, the one-complex-variable approach [21] can be used to simplify the analysis.

Once the complex potential $\mathbf{f}(z)$ and $g(z)$ are obtained, all the fields can be determined by Eqs. (4, 5, 24, 25).

\subsection{Mapping function}

A crack originating from the edge of a half elliptical hole in the half thermopiezoelectric plane under the remote thermal-mechanical-electric loadings is shown in Fig. 1. $x_{1}-x_{2}$ is the anisotropic plane, the poling direction is along the positive axis $x_{1}, L$ is the crack length, and $a / b$ is the length of semi-major/semi-minor.

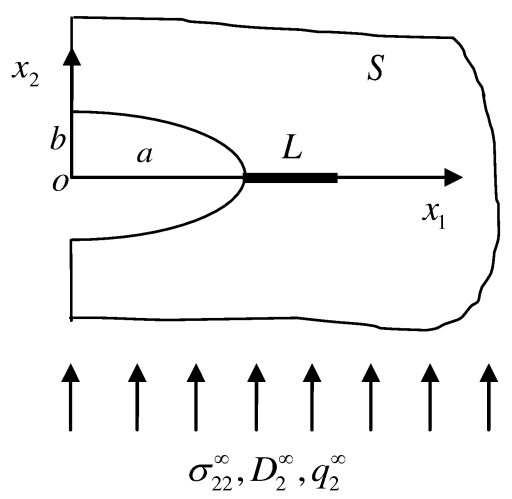

Fig. 1. Crack originating from a half elliptical hole in the half piezoelectric plane

The mapping function, which maps the outside of the elliptical hole and cracks into the interior of a unit circle in the $z_{1}$-plane, has the form as:

$$
z_{\alpha}=\omega_{\alpha}\left(z_{1}\right)=R_{\alpha} \mu\left(z_{1}\right)+R_{\alpha} m_{\alpha} \mu\left(z_{1}\right) \quad(\alpha=t, 1,2,3,4),
$$

which is given by Guo [11, 12], and:

$$
\begin{aligned}
& \mu\left(z_{1}\right)=\frac{1}{4 \zeta}\left\{\varepsilon_{1}\left(1+z_{1}\right)^{2}+\varepsilon_{2}\left(1-z_{1}\right)^{2}+\right. \\
& \left.\sqrt{\left(\varepsilon_{1}^{2}-1\right)\left(1+z_{1}\right)^{4}+2\left(\varepsilon_{1} \varepsilon_{2}+1\right)\left(1-z_{1}^{2}\right)^{2}+\left(\varepsilon_{2}^{2}-1\right)\left(1-z_{1}\right)^{4}}\right\},
\end{aligned}
$$




$$
\begin{gathered}
R_{\alpha}=\left(a-i \mu_{\alpha} b\right) / 2, \\
m_{\alpha}=\left(a+i \mu_{\alpha} b\right) /\left(a-i \mu_{\alpha} b\right) .
\end{gathered}
$$

$\varepsilon$ is a real parameter, which can be expressed as:

$$
\varepsilon_{i}=\frac{\left(a+L_{i}\right)^{2}+b^{2}+a b+\left(a+L_{i}\right) \sqrt{L_{i}^{2}+2 a L_{i}+b^{2}}}{(a+b)\left(a+L_{i}+\sqrt{L_{i}^{2}+2 a L_{i}+b^{2}}\right)},(i=1,2) .
$$

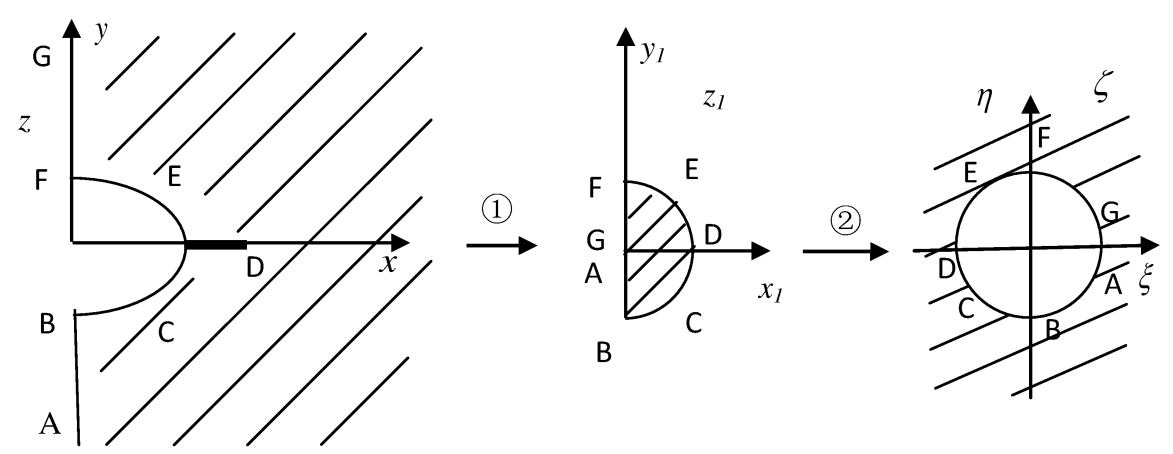

Fig. 2. Process of the conformal transformation

In this case, $i=2, L_{1}=L_{2}=L$. Eq. (26) also maps the imaginary axis into the imaginary axis in the $z_{1}$-plane. That is, Eq. (26) maps the right physics plane into the interior of a right unit circle in the $z_{1}$-plane (Fig. 2, process 1). The mapping function, which maps the interior of a right unit circle in the $z_{1}$-plane into the outside of a unit circle in the $\zeta$-plane, can be written as (Fig. 2, process 2):

$$
z_{1}=f(\zeta)=-\frac{\zeta+1}{\zeta-1}+\sqrt{\left(\frac{\zeta+1}{\zeta-1}\right)^{2}+1} .
$$

Eqs. (26) and (27) are the mapping functions which map the outside region of the elliptical hole and the crack into the outside region of a unit circle in the $\zeta$-plane. The correspondence of the points between different planes can be seen in Fig. 2.

For a given point $z$ at the boundary of the hole and the crack, one can obtain five points $z_{\alpha}(\alpha=t, 1,2,3,4)$ in the $z_{\alpha}$-plane, this mapping function 
can map these five points into one unique point on the unit circle in the $\zeta$-plane, and $\zeta=-1$ corresponds to the crack tip.

The mapping function Eqs. (26) and (27) can be re-written in the form of Laurent series as:

$$
\omega(\zeta)=\sum_{m=-\infty}^{1} b_{m} \zeta^{m},
$$

where $b_{m}=\frac{1}{2 \pi i} \oint \frac{\omega(\zeta)}{\zeta^{m+1}} d \zeta,(m>1), b_{-1}=R_{\alpha}\left(\frac{\varepsilon_{1}+\varepsilon_{2}}{2}-\frac{2}{\varepsilon_{1}+\varepsilon_{2}}\right)+$ $R_{\alpha} m_{\alpha} \frac{\varepsilon_{1}+\varepsilon_{2}}{2}, b_{1}=R_{\alpha} \frac{\varepsilon_{1}+\varepsilon_{2}}{2}$, and $b_{0}=R_{\alpha}\left(\varepsilon_{1}-\varepsilon_{2}\right)$.

\section{Theoretical analysis}

\subsection{Complex potential for the temperature field}

The thermal potential can be written as

$$
g^{\prime}(z)=a_{1} z+g_{0}^{\prime}(z)
$$

where $a_{1}$ is a constant to be determined, $g_{0}^{\prime}(z)$ is a holomorphic function outside the hole, and $g_{0}^{\prime}(\infty)=a_{2}, a_{2}$ is a constant corresponding an uniform temperature field and thus, it can be neglected without loss in generality.

In order to determine $a_{1}$, one can take the limiting $z \rightarrow \infty$ in Eqs. (4)and (5), and gives:

$$
\begin{aligned}
& q_{1}^{\infty}=2 \operatorname{Re}\left[i \mu_{t} \kappa_{t} g^{\prime \prime}(\infty)\right]=2 \operatorname{Re}\left[i \mu_{t} \kappa_{t} a_{1}\right], \\
& q_{2}^{\infty}=-2 \operatorname{Re}\left[i \kappa_{t} g^{\prime \prime}(\infty)\right]=-2 \operatorname{Re}\left[i \kappa_{t} a_{1}\right] .
\end{aligned}
$$

From Eqs. (30) and (31), $a_{1}$ can be determined as:

$$
a_{1}=\frac{q_{1}^{\infty}+\overline{\mu_{t}} q_{2}^{\infty}}{i \kappa_{t}\left(\mu_{t}-\overline{\mu_{t}}\right)} .
$$

Substituting Eq. (29) into Eq. (10) yields:

$$
2 \operatorname{Re}\left[i \kappa_{t} a_{1} z+i \kappa_{t} g_{0}^{\prime}(z)\right]=0
$$

which can be re-written as:

$$
g_{0}^{\prime}(z)-\overline{g_{0}^{\prime}(z)}=\overline{a_{1} z}-a_{1} z .
$$


In the $\zeta$-plane on the surface of the hole and the crack, Eq. (34) becomes:

$$
\frac{g_{0}^{\prime}(\sigma)}{\omega^{\prime}(\sigma)}-\frac{\overline{g_{0}^{\prime}(\sigma)}}{\overline{\omega^{\prime}(\sigma)}}=\overline{a_{1} \omega(\sigma)}-a_{1} \omega(\sigma)
$$

where $g_{0}^{\prime}(\zeta)=g_{0}^{\prime}(\omega(\zeta))$ is defined.

From Eq. (28), it can be found that $\omega^{\prime}(\zeta)\left(\omega^{\prime}(\infty) \neq 0\right)$ is a holomorphic function at infinity. Since $g_{0}^{\prime}(\zeta)$ is also a holomorphic function outside the unit circle, $g_{0}^{\prime}(\zeta) / \omega^{\prime}(\zeta)$ is a holomorphic function, thus its conjugate function $\overline{g_{0}^{\prime}(\zeta)} / \overline{\omega^{\prime}(\zeta)}\left(\omega^{\prime}(\zeta) \neq 0\right)$ is holomorphic inside the unit circle.

Taking Cauchy integrals at the two sides of Eq. (35) gives [22]:

$$
a_{2}-\frac{g_{0}^{\prime}(\zeta)}{\omega^{\prime}(\zeta)}=-\overline{a_{1}} b_{1} \zeta^{-1}+a_{1}\left(\omega(\zeta)-b_{1} \zeta-b_{0}\right)
$$

which leads to:

$$
g_{0}^{\prime}(\zeta)=a_{2} \omega^{\prime}(\zeta)+\overline{a_{1}} b_{1} \omega^{\prime}(\zeta) \zeta^{-1}-a_{1} \omega^{\prime}(\zeta)\left(\omega(\zeta)-b_{1} \zeta-b_{0}\right) .
$$

Integration to Eq. (37),

$$
\begin{aligned}
g_{0}(\zeta)= & a_{2} \omega(\zeta)+\overline{a_{1}} b_{1}\left(b_{1} \ln \zeta-\sum_{j=1}^{n} j b_{j} \frac{\zeta^{-j-1}}{-j-1}\right) \\
& +a_{1} \sum_{j=1}^{n} b_{-j}\left(\sum_{m=-1,(m=-1 \& i \neq 1)}^{n} m b_{-m} \frac{\zeta^{-m-j}}{-m-j}\right)-a_{1} b_{1} b_{-1} \ln \zeta \\
= & a_{2} \omega(\zeta)+a_{3} \ln \zeta+g_{10}(\zeta),
\end{aligned}
$$

where,

$$
\begin{gathered}
a_{3}=\overline{a_{1}} b_{1}^{2}-a_{1} b_{-1} b_{1}, \\
g_{10}(\zeta)=\overline{a_{1}} b_{1}\left(\sum_{j=1}^{n} j b_{j} \frac{\zeta^{-j-1}}{j+1}\right)+a_{1} \sum_{j=1}^{n} b_{-j}\left(\sum_{m=-1,(m=-1 \& j \neq 1)}^{n} m b_{-m} \frac{\zeta^{-m-j}}{-m-j}\right) .
\end{gathered}
$$

Returning to the $z$-plane, Eq.(37) becomes:

$$
g_{0}^{\prime}(z)=a_{2}+\overline{a_{1}} b_{1} \zeta(z)^{-1}-a_{1}\left(z-b_{1} \zeta(z)-b_{0}\right) .
$$


Finally, the thermal complex potential can be expressed as:

$$
g(z)=\frac{1}{2} a_{1} z^{2}+a_{2} z+a_{3} \ln (\zeta(z))+g_{10}(z) .
$$

Inserting Eq. (40) into Eqs. (4) and (5), the heat flux field can be obtained. Due to the complication of Eq. (38), it is not easy to get a clear form of heat flux. However, it can be seen from Eqs. (29) and (38) that $g^{\prime \prime}(\infty)=a_{1}$, which is related to the remote heat flux, and $g^{\prime \prime}(0)=\infty$, which means the heat flux has singularity at the crack tip, the type of singularity will be discussed in Section 3.3, and the numerical results of the heat flux can be seen in Section 4.1.

\subsection{Complex potentials for the mechanical-electric field}

Based on Eq. (40), the complex potentials for mechanical and electric field can be expressed as:

$$
\mathbf{f}(z)=\frac{1}{2} \mathbf{a}_{4} z^{2}+\mathbf{a}_{5} z+\mathbf{a}_{6} \ln (\zeta(z))+\mathbf{f}_{0}(z),
$$

where $\mathbf{a}_{4}, \mathbf{a}_{5}$ and $\mathbf{a}_{6}$ are three constant vectors to be found, and $\mathbf{f}_{0}(z)$ is a holomorphic function outside the hole and the crack.

Taking the limitation $z \rightarrow \infty$ in Eqs. (24) and (25), one has:

$$
\begin{aligned}
& \mathbf{u}_{, 1}^{\infty}=2 \operatorname{Re}\left[\mathbf{A}\left(\mathbf{a}_{4} z+\mathbf{a}_{5}\right)+\mathbf{c}\left(a_{1} z+a_{2}\right)\right], \\
& \boldsymbol{\varphi}_{, 1}^{\infty}=2 \operatorname{Re}\left[\mathbf{B}\left(\mathbf{a}_{4} z+\mathbf{a}_{5}\right)+\mathbf{d}\left(a_{1} z+a_{2}\right)\right],
\end{aligned}
$$

where $\mathbf{u}_{11}^{\infty}=\left(\gamma_{11}^{\infty}, \gamma_{12}^{\infty}+\omega_{3}^{\infty}, 2 \gamma_{13}^{\infty},-E_{1}^{\infty}\right)^{T}, \varphi_{11}^{\infty}=\left(\sigma_{21}^{\infty}, \sigma_{22}^{\infty}, \sigma_{23}^{\infty}, D_{2}^{\infty}\right)^{T}$. It is obvious, that the stress, strain and electric displacement are bounded at infinity, then Eq. (42) gives:

$$
\begin{aligned}
& 2 \operatorname{Re}\left[\mathbf{A a}_{4} z+\mathbf{c} a_{1} z\right]=\mathbf{0}, \\
& 2 \operatorname{Re}\left[\mathbf{B a}_{4} z+\mathbf{d} a_{1} z\right]=\mathbf{0},
\end{aligned}
$$

and

$$
\begin{aligned}
& 2 \operatorname{Re}\left[\mathbf{A a}_{5}+\mathbf{c} a_{2}\right]=\mathbf{u}_{, 1}^{\infty}, \\
& 2 \operatorname{Re}\left[\mathbf{B} \mathbf{a}_{5}+\mathbf{d} a_{2}\right]=\boldsymbol{\varphi}_{, 1}^{\infty} .
\end{aligned}
$$

From Eq. (44), $\mathbf{a}_{5}$ can be determined by using Eq. (21):

$$
\mathbf{a}_{5}=\mathbf{B}^{T} \mathbf{u}_{, 1}^{\infty}+\mathbf{A}^{T} \varphi_{, 1}^{\infty}-\mathbf{B}^{T}\left(\mathbf{c} a_{2}+\overline{\mathbf{c} a_{2}}\right)-\mathbf{A}^{T}\left(\mathbf{d} a_{2}+\overline{\mathbf{d} a_{2}}\right) .
$$


In Eq. (43), it can be found, that the complex functions $\left[\mathbf{A a}_{4} z+\mathbf{c} a_{1} z\right]$ and $\left[\mathbf{B a}_{4} z+\mathbf{d} a_{1} z\right]$ stand for the uniform heat flow in an infinite medium without holes, and they will not produce stress or strain, thus they can be cut out in the equations. Under this assumption, Eqs. (40) and (41) becomes:

$$
\begin{gathered}
g(z)=a_{2} z+a_{3} \ln (\zeta(z))+g_{10}(z), \\
\mathbf{f}(z)=\mathbf{a}_{5} z+\mathbf{a}_{6} \ln (\zeta(z))+\mathbf{f}_{0}(z) .
\end{gathered}
$$

In Eqs. (46) and $(47), \ln (\zeta(z))$ is a multi-value item. In order to determine the coefficient $\mathbf{a}_{6}$, the condition of single - valued displacement and the equilibrium of mechanical and electrical is used:

$$
\oint_{\Gamma_{n}} \mathbf{u}_{, 1} d z=0, \quad \oint_{\Gamma_{n}} \boldsymbol{\varphi}_{, 1} d z=0
$$

where $\Gamma_{n}$ stands for a clockwise closed-contour encircling the hole, and:

$$
\begin{gathered}
\mathbf{u}_{, 1}=2 \operatorname{Re}\left[\mathbf{A f}^{\prime}(z)+\mathbf{c} g^{\prime}(z)\right], \\
\boldsymbol{\varphi}_{, 1}=2 \operatorname{Re}\left[\mathbf{B} \mathbf{f}^{\prime}(z)+\mathbf{d} g^{\prime}(z)\right] .
\end{gathered}
$$

Substituting Eqs. (49) and (50) into Eq. (48), that:

$$
\begin{aligned}
& \mathbf{A} \mathbf{a}_{6}+\mathbf{c} a_{3}-\overline{\left(\mathbf{A} \mathbf{a}_{6}+\mathbf{c} a_{3}\right)}=0, \\
& \mathbf{B a}_{6}+\mathbf{d} a_{3}-\overline{\left(\mathbf{B a _ { 6 } + \mathbf { d } a _ { 3 } )}\right.}=0 .
\end{aligned}
$$

By using Eq. (21), $\mathbf{a}_{6}$ can be obtained from Eqs. (51)and (52),

$$
\mathbf{a}_{6}=\mathbf{B}^{T}\left(\overline{\mathbf{c} a_{3}}-\mathbf{c} a_{3}\right)+\mathbf{A}^{T}\left(\overline{\mathbf{d} a_{3}}-\mathbf{d} a_{3}\right) .
$$

Mechanical and electric condition along the boundary requires:

$$
2 \operatorname{Re}[\mathbf{B f}(z)+\mathbf{d} g(z)]=\int_{s} \mathbf{t}_{s} d s, \quad \mathbf{t}_{s}=\left(t_{1}, t_{2}, t_{3}, D_{n}\right)^{T} .
$$

Based on the assumption, that the crack and the hole are both electrically impermeable and mechanically free, Eq. (54) can be reduced to:

$$
2 \operatorname{Re}[\mathbf{B f}(z)+\mathbf{d} g(z)]=0 .
$$


In the $\zeta$-plane, Eq. (55) becomes:

$$
2 \operatorname{Re}[\mathbf{B f}(\zeta)+\mathbf{d} g(\zeta)]=0 .
$$

Eqs. (46) and (47) become:

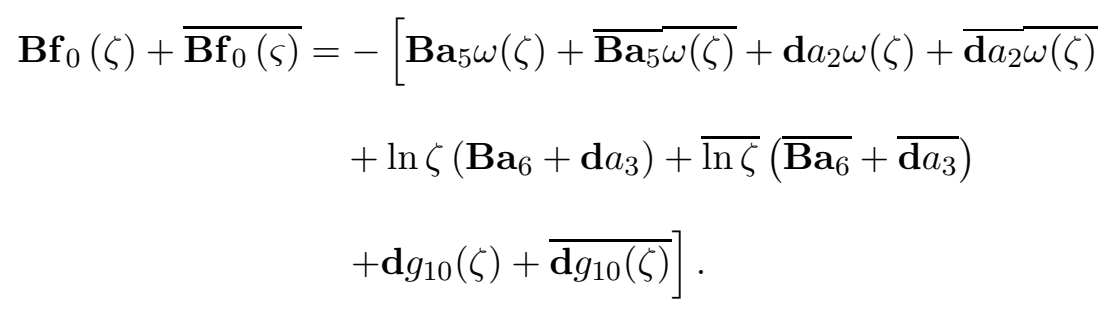$$
\text { Substituting Eqs. (57) and (58) into Eq. (56) yields: }
$$

At the boundary of the hole and the crack, Eq. (59) becomes:

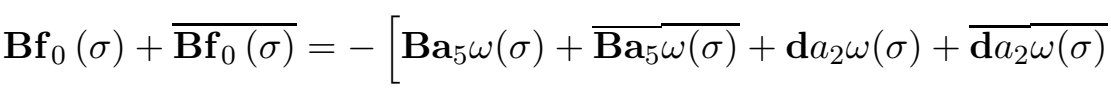

$$
\begin{aligned}
& \left.+\mathbf{d} g_{10}(\sigma)+\overline{\mathbf{d} g_{10}(\sigma)}\right] .
\end{aligned}
$$

According to Eq. (28), $\omega(\zeta)$ is a holomorphic function outside the circle, except at infinity at which it has singular principle part $b_{1} \zeta+b_{0}$, and $\overline{\omega(\zeta)}$ is a holomorphic function in the circle except the zero point, at which it has singular principle part $b_{1} \zeta^{-1} \cdot g_{10}(\zeta)$ is a holomorphic function outside the unit circle, and $\overline{g_{10}(\zeta)}$ is a holomorphic function inside the unit circle.

Taking Cauchy integrals at the two sides of Eq. (60) gives [22]:

$(61) \mathbf{B f}_{0}(\zeta)=-\boldsymbol{\varphi}_{, 1}^{\infty} b_{1} \zeta^{-1}-\left(\mathbf{B a}_{5}+\mathbf{d} a_{2}\right)\left(\omega(\zeta)-b_{1} \zeta-b_{0}-b_{1} \zeta^{-1}\right)-\mathbf{d} g_{10}(\zeta)$, where Eq. (44) has been used. From Eq. (61), one can get:

$$
\mathbf{B f}_{0}^{\prime}(\zeta)=\boldsymbol{\varphi}_{, 1}^{\infty} b_{1} \zeta^{-2}-\left(\mathbf{B a}_{5}+\mathbf{d} a_{2}\right)\left(\omega^{\prime}(\zeta)-b_{1}+b_{1} \zeta^{-2}\right)-\mathbf{d} g_{10}^{\prime}(\zeta) .
$$

At the crack tip, $\zeta=-1$ and Eq. (62) gives:

$$
\mathbf{B} \mathbf{f}_{0}^{\prime}(-1)=\varphi_{, 1}^{\infty} b_{1}-\mathbf{d} g_{10}^{\prime}(-1)
$$


with Eq. (63), the FIF can be calculated in the next section.

Once $\mathbf{f}_{0}(\zeta)$ is obtained, the expression of stresses and electric displacement can be derived by:

$$
\begin{gathered}
\boldsymbol{\varphi}_{, 1}=\boldsymbol{\varphi}_{, 1}^{\infty}+2 \operatorname{Re}\left[\frac{\mathbf{B} \mathbf{a}_{6}+\mathbf{d} a_{3}}{\omega^{\prime}(\zeta) \zeta}+\frac{\mathbf{B} \mathbf{f}_{0}^{\prime}(\zeta)+\mathbf{d} g_{10}^{\prime}(\zeta)}{\omega^{\prime}(\zeta)}\right], \\
\boldsymbol{\varphi}_{, 2}=\boldsymbol{\varphi}_{, 2}^{\infty}+2 \operatorname{Re}\left[\mu \frac{\mathbf{B} \mathbf{a}_{6}+\mathbf{d} a_{3}}{\omega^{\prime}(\zeta) \zeta}+\mu \frac{\mathbf{B} \mathbf{f}_{0}^{\prime}(\zeta)+\mathbf{d} g_{10}^{\prime}(\zeta)}{\omega^{\prime}(\zeta)}\right] .
\end{gathered}
$$

Eqs. (64) and (65) have the same form with the expression of the stresses and electric displacement in the full plane, which means the solution of the half plane is identical to the solution of the full plane, under the same condition. The distribution of the stress and the electric displacement can be found in Ref. [15].

\subsection{Field intensity factors}

The vector of FIF can be defined as:

$$
\mathbf{k}=\left(k_{\mathrm{II}}, k_{\mathrm{I}}, k_{\mathrm{III}}, k_{D}\right)^{T}=\lim _{z \rightarrow z_{1}} \sqrt{2 \pi\left(z-z_{1}\right)} \varphi_{, 1},
$$

where $z_{1}=a+L_{1}$.

Inserting $\boldsymbol{\varphi}_{, 1}$ into Eq. (66) results in:

$$
\mathbf{k}=\operatorname{Re}\left[\lim _{z \rightarrow z_{1}} \sqrt{2 \pi\left(z-z_{1}\right)} 2\left[\mathbf{B f}^{\prime}(z)+\mathbf{d} g^{\prime}(z)\right]\right] .
$$

In the $\zeta$-plane, Eq. (67) becomes:

(68)

$$
\mathbf{k}=\operatorname{Re}\left[2 \sqrt{2 \pi} \lim _{\zeta \rightarrow-1} \sqrt{\omega(\zeta)-\omega(-1)}\left[\frac{\mathbf{B} \mathbf{a}_{6}+\mathbf{d} a_{3}}{\zeta \omega^{\prime}(\zeta)}+\frac{\mathbf{B} \mathbf{f}_{0}^{\prime}(\zeta)+\mathbf{d} g_{10}^{\prime}(\zeta)}{\omega^{\prime}(\zeta)}\right]\right] .
$$

In addition, it can be shown from the requirement of filed singularity at the crack tips, that $\omega^{\prime}(-1)=0$. Here, $L \neq 0$. Thus, by using the L' Hospital rule, Eq. (68) becomes:

$$
\mathbf{k}=\frac{2 \sqrt{\pi}}{\sqrt{\omega^{\prime \prime}(-1)}} \operatorname{Re}\left[\mathbf{B} \mathbf{a}_{6}+\mathbf{d} a_{3}+\mathbf{B} \mathbf{f}_{0}^{\prime}(-1)+\mathbf{d} g_{10}^{\prime}(-1)\right]
$$


Inserting $\mathbf{a}_{6}, a_{3}, \mathbf{f}_{0}^{\prime}(1)$ and $g_{10}^{\prime}(1)$ into Eq. (69) yields:

$$
\mathbf{k}=\frac{2 \sqrt{\pi}}{\sqrt{\omega^{\prime \prime}(-1)}} \operatorname{Re}\left[b_{1} \boldsymbol{\varphi}_{, 1}^{\infty}+\mathbf{B} \mathbf{a}_{6}+\mathbf{d} a_{3}\right]
$$

It can be seen, that Eq. (70) has the exact same form with the Eq (62) in Ref. [15], and Eq. (70) can be reduced to the exact result of Eq. (52) in Guo et al. [11], if there is no heat flux.

In Eq. (70), item $b_{1} \varphi_{, 1}^{\infty}$ stands for the contribution of the applied mechanical-electric loadings to all the filed singularities, while items $\mathbf{B a}_{6}+\mathbf{d} a_{3}$ stand for the contribution of heat loading to all the filed singularities. If $L=0$, Eq. (70) will possess the same form with the Eqs. (131-133) in Gao et al. [6].

For this permeable crack case, the FIF is depended on the size of the shape and the corresponding external loadings. For the impermeable crack, according to Refs. [6] and [12], the stress intensity factor is still dependent on the size of the shape and the mechanical loading, but the electric displacement intensity factor is no longer dependent on the electric loading, but depends on the mechanical loading, which means the electric displacement intensity factor is induced by the piezoelectric effect.

Since the FIF result Eq. (70) is acceptable, based on the FIF definition Eq. (66), the stress and electric displacement Eqs. (64) and (65) obtained from complex potential function Eq. (62) have the singularity of $r^{-0.5}$ at the crack tip. In addition, the heat flux complex potential function has the same form with the complex potential function Eq. (62), which means the heat flux also has the singularity of $r^{-0.5}$ at the crack tip.

To be simplified, the new variables $g=\mathbf{k} /\left(\varphi_{1}^{\infty} \sqrt{\pi a_{e}}\right)$ and $a_{e}=a+L$ are introduced in the following numerical examples:

\section{Numerical examples}

Consider a transversely isotropic piezoelectric medium, in which $X_{1}-$ $X_{2}$ plane is assumed to be the isotropic plane, and the positive $X_{3}$-axis is the polling direction. Take cadmium selenide as a model material with the following 
material constants $[6,15]$ :

$$
\begin{aligned}
& c_{11}=74.1 \times 10^{9} \mathrm{Nm}^{-2}, \quad c_{12}=45.2 \times 10^{9} \mathrm{Nm}^{-2}, \quad c_{13}=39.3 \times 10^{9} \mathrm{Nm}^{-2}, \\
& c_{33}=83.6 \times 10^{9} \mathrm{Nm}^{-2}, \quad c_{44}=13.2 \times 10^{9} \mathrm{Nm}^{-2} . \\
& e_{31}=-0.16 \mathrm{Cm}^{-2}, \quad e_{33}=0.347 \mathrm{Cm}^{-2}, \quad e_{15}=-0.138 \mathrm{Cm}^{-2} . \\
& \varepsilon_{11}=82.6 \times 10^{-12} \mathrm{CV}^{-1} \mathrm{~m}^{-1}, \quad \varepsilon_{33}=90.3 \times 10^{-12} \mathrm{CV}^{-1} \mathrm{~m}^{-1} . \\
& \beta_{11}=0.621 \times 10^{6} \mathrm{NK}^{-1} \mathrm{~m}^{-2}, \quad \beta_{33}=0.551 \times 10^{6} \mathrm{NK}^{-1} \mathrm{~m}^{-2} . \\
& \tau_{3}=-2.94 \times 10^{-6} \mathrm{CK}^{-1} \mathrm{~m}^{-2}, \quad \lambda_{11}=\lambda_{22}=10^{-6} \mathrm{Wm}^{-1} \mathrm{~K}^{-1}, \quad \lambda_{33}=1.5 \lambda_{11} .
\end{aligned}
$$

Where $\mathrm{N}$ is the force in Newtons, $\mathrm{C}$ is the charge in coulombs, $\mathrm{V}$ is the electric potential in volts, $\mathrm{m}$ is the length in meters, $\mathrm{K}$ is the temperature in Kelvins, and $\mathrm{W}$ is the energy in Watts.

For convenience, the $X_{1}-X_{3}$ plane is relabelled as the $x_{1}-x_{2}$ plane in the following analysis, and studies are focused on the $2 \mathrm{D}$ problem in the $x_{1}-x_{2}$ plane. For this case, the out-of plane displacement dose not couple with the in-plane displacement and the electric potential, and thus the matrices $\mathbf{S}, \mathbf{R}$ and $\mathbf{W}$ degenerate into $3 \times 3$ ones:

$$
\mathbf{S}=\left[\begin{array}{ccc}
c_{11} & 0 & 0 \\
0 & c_{44} & e_{15} \\
0 & e_{15} & -\varepsilon_{11}
\end{array}\right], \quad \mathbf{R}=\left[\begin{array}{ccc}
0 & c_{13} & e_{31} \\
c_{44} & 0 & 0 \\
e_{15} & 0 & 0
\end{array}\right], \quad \mathbf{W}=\left[\begin{array}{ccc}
c_{44} & 0 & 0 \\
0 & c_{33} & e_{33} \\
0 & e_{33} & -\varepsilon_{33}
\end{array}\right]
$$

and

$$
\beta_{1}=\left(\beta_{11}, 0,0\right)^{T}, \quad \beta_{2}=\left(0, \beta_{33}, \tau_{3}\right)^{T} .
$$

The eigenvalues obtained from the given constants are:

$$
\mu_{1}=1.8267 i, \quad \mu_{2}=0.8303 i, \quad \mu_{3}=0.5941 i, \quad \mu_{t}=0.816 i .
$$

$\mathbf{A}$ and $\mathbf{B}$ matrixes are:

$$
\begin{gathered}
\mathbf{A}=\left[\begin{array}{ccc}
3.1992 \times 10^{-6}(1-i) & 1.5953 \times 10^{-6}(-1+i) & 1.7736 \times 10^{-6}(-1-i) \\
1.0843 \times 10^{-6}(1+i) & 2.0528 \times 10^{-6}(-1-i) & 3.8023 \times 10^{-6}(1-i) \\
1.4388 \times 10^{4}(1+i) & 5.7446 \times 10^{4}(1+i) & 2.5718 \times 10^{4}(-1+i)
\end{array}\right], \\
\mathbf{B}=\left[\begin{array}{ccc}
8.9466 \times 10^{4}(1+i) & 5.2508 \times 10^{4}(-1-i) & 6.7649 \times 10^{4}(1-i) \\
4.9878 \times 10^{4}(-1+i) & 6.3241 \times 10^{4}(1-i) & 1.1386 \times 10^{5}(1+i) \\
1.1740 \times 10^{-6}(1-i) & 5.1536 \times 10^{-6}(1-i) & 2.4475 \times 10^{-6}(1+i)
\end{array}\right]
\end{gathered}
$$




\subsection{Distribution of the heat flux}

Take $q_{1}^{\infty}=0, q_{2}^{\infty}=1 \mathrm{Wm}^{-2}, a=0.01 \mathrm{~m}$. The distribution of the heat flux $q_{2}$ along the axis $x_{1}$ is shown in Fig. 3. It can be seen, that the heat flux trends to $q_{2}^{\infty}$ at infinity, and trends to infinity at the crack tip. Besides, the heat flux $q_{2}$ gets higher as the crack length grows.

Let $a=b=0.01 \mathrm{~m}$, and the elliptical hole becomes a circular hole, which is more common in practice. The distribution of the heat flux along the axis $x_{1}$ is given in Fig. 4. It can be seen, that the distribution of the heat flux in Figs 3 and 4 are similar; the heat flux in Fig. 4 is a little smaller than the one in Fig. 3 under the same crack length, which means the circular hole can reduce the distribution of heat flux at the crack tip, compared with the elliptical hole $(b / a=0.5)$.

\subsection{Distribution of the stress and the electric displacement}

Let $L=0 \mathrm{~m}$, and $a=0.01 \mathrm{~m} b=0 \mathrm{~m}, 0.005 \mathrm{~m}, 0.01 \mathrm{~m}, 0.02 \mathrm{~m}$, which corresponding to the pure crack, elliptical hole, circular hole and the elliptical hole, respectively. $q_{2}^{\infty}=1 \mathrm{Wm}^{-2}, \sigma_{22}^{\infty}=100 \mathrm{Mpa}, D_{2}^{\infty}=2 e-3 \mathrm{C} / \mathrm{m}^{2}$. The distribution of the stress and the electric displacement along the axis $x_{1}$ for the edge elliptical hole problem are given, as shown in Figs 5 and 6. It can be seen, the stress and the electric displacement trend to external loads at the infinity. Besides, the stress and the electric displacement increase as $b$ decreases at the same place.

\subsection{Distribution of the field intensity factors}

Let $q_{2}^{\infty}=1 \mathrm{Wm}^{-2}, \sigma_{22}^{\infty}=100 \mathrm{MPa}, D_{2}^{\infty}=1 \mathrm{C} / \mathrm{m}^{-2}, a=0.01 \mathrm{~m}$, and $b=0.5 a$, the distribution of equivalent FIF with crack length are shown in Fig. 7. It can be found, that the equivalent FIF trend to constant 1 as the crack length grows. When the crack length is long enough, the effect of the hole size on the crack tip can be ignored.

The distribution of the equivalent FIF with the $b / a$ are shown in Fig. 8 . In this case, let $q_{2}^{\infty}=1 \mathrm{Wm}^{-2}, \sigma_{22}^{\infty}=100 \mathrm{MPa}, D_{2}^{\infty}=1 \mathrm{C} / \mathrm{m}^{-2}, L=0.01 \mathrm{~m}$ and $a=0.01 \mathrm{~m}$, it can be seen that equivalent FIF decreases as the $b$ increases. When $b=0 \mathrm{~m}$, the equivalent FIF becomes to a constant 1, which is acceptable. This phenomenon means the hole at the side of a crack can reduce the FIF, if the crack already exists. The larger the hole size, the more obvious is the effect. However, this conclusion is only for the right crack tip. When $b$ exceeds a certain value, for example $b \gg a$, the singularity at the upper and lower ends of the point of the ellipse may be even bigger than the right crack tip. So, basically, circular hole is the best option to reduce the singularity at the crack 


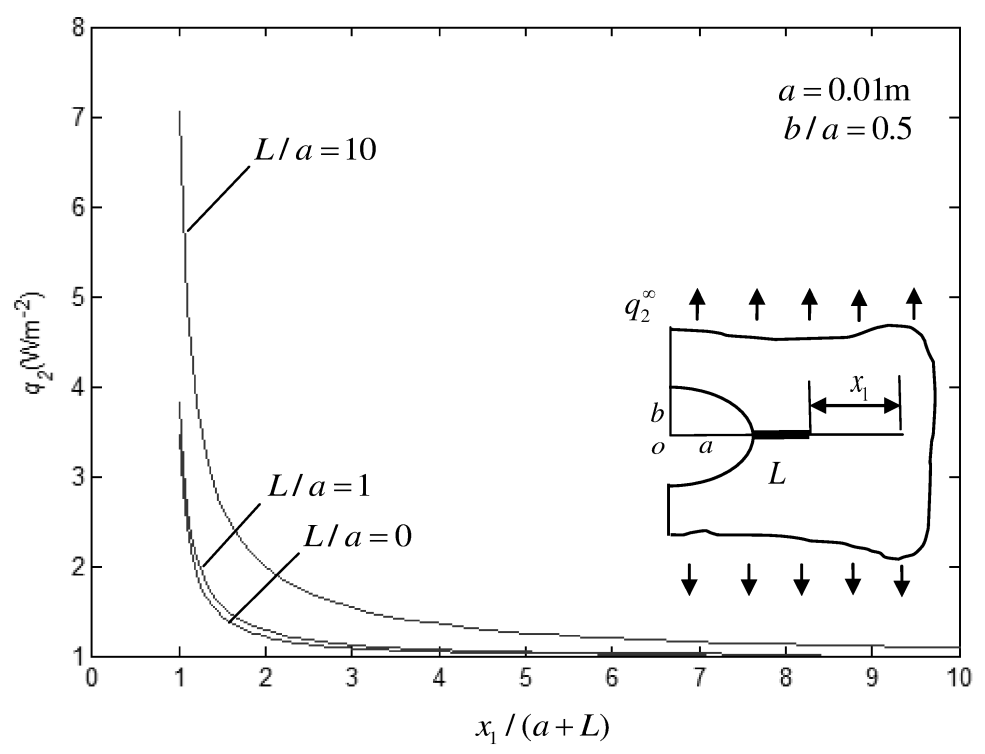

Fig. 3. Distribution of the heat flux $q_{2}$ along axis $x_{1}$ (elliptical hole)

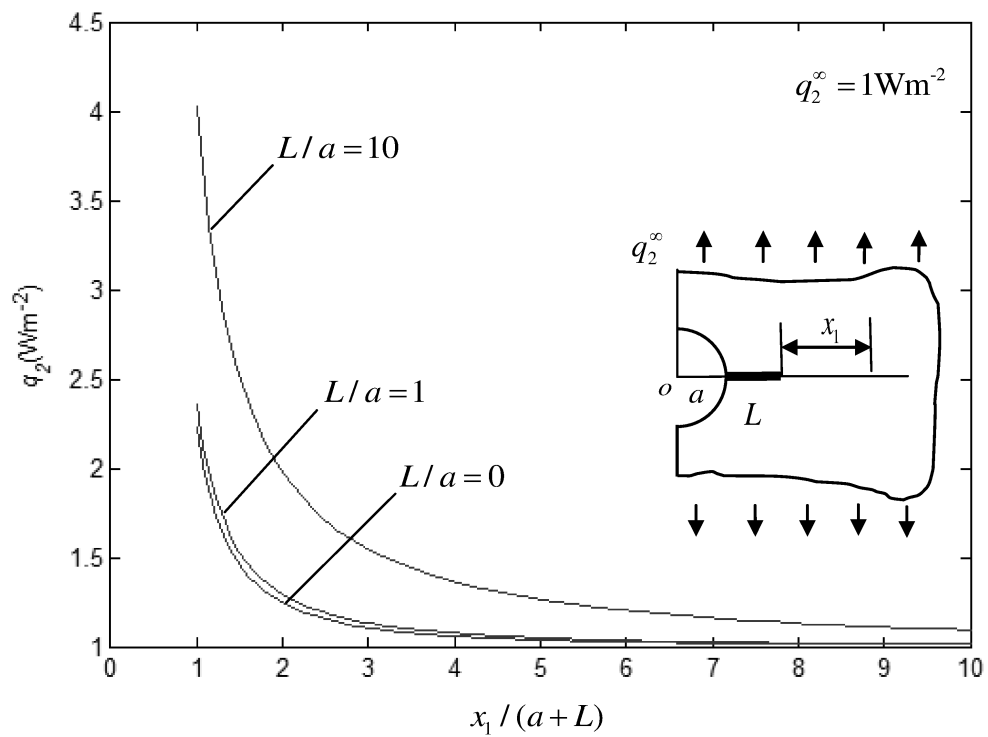

Fig. 4. Distribution of the heat flux $q_{2}$ along axis $x_{1}$ (circular hole) 


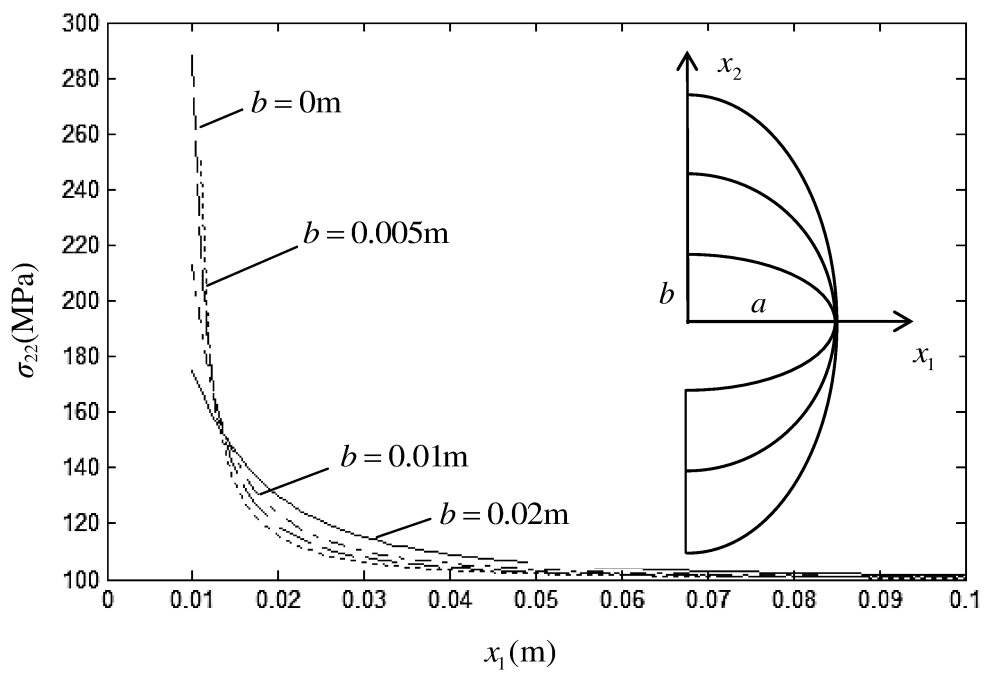

Fig. 5. Distribution of the stress $\sigma_{22}$ along axis $x_{1}$

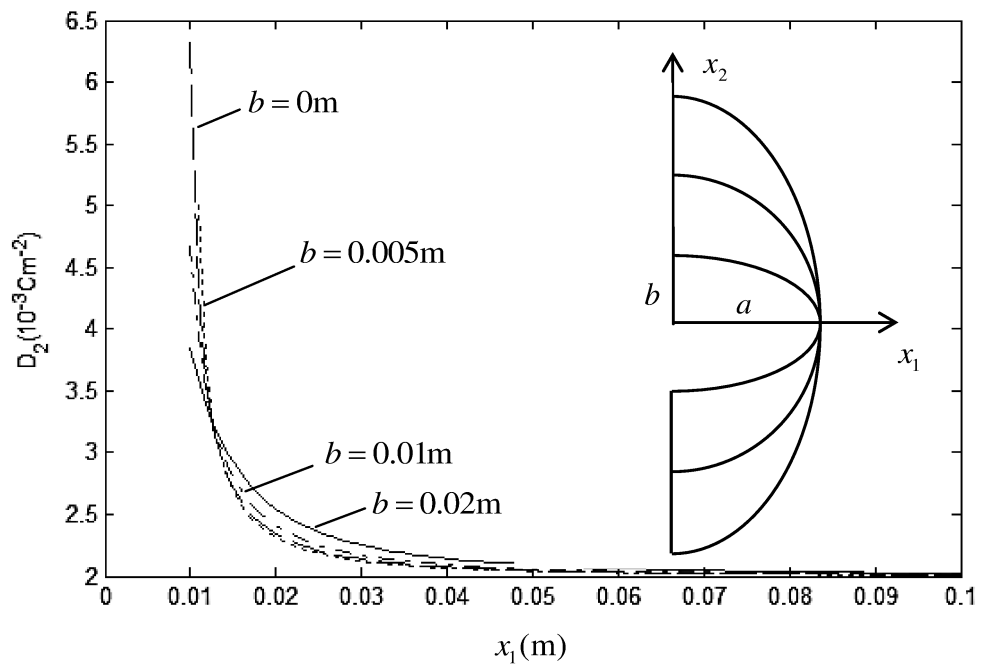

Fig. 6. Distribution of the electric displacement $D_{2}$ along axis $x_{1}$ 


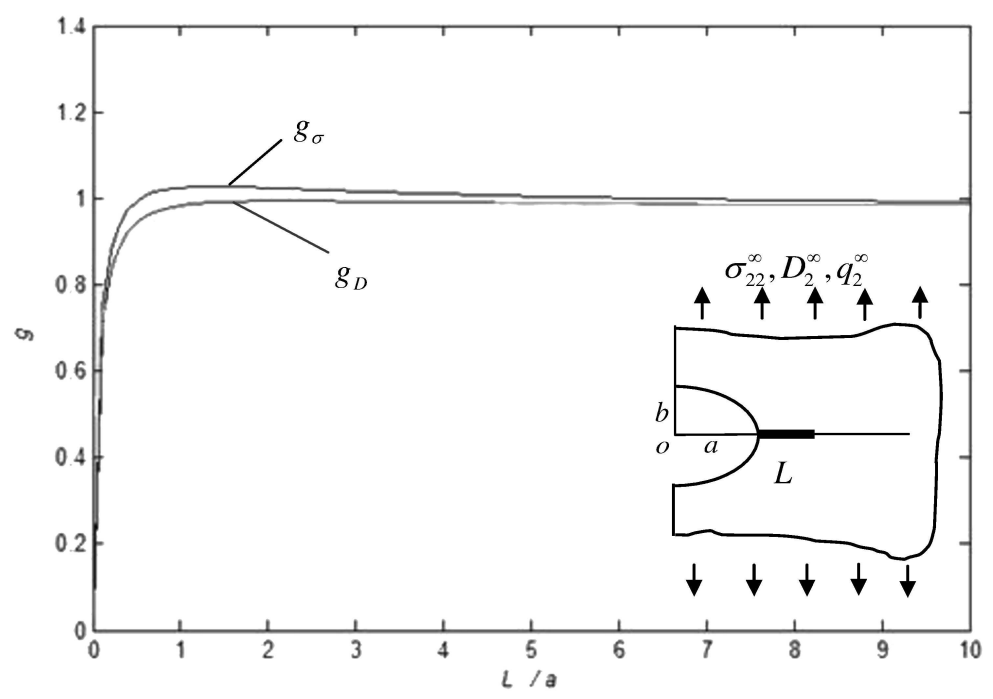

Fig. 7. Distribution of the equivalent fields intensity factors with the crack length

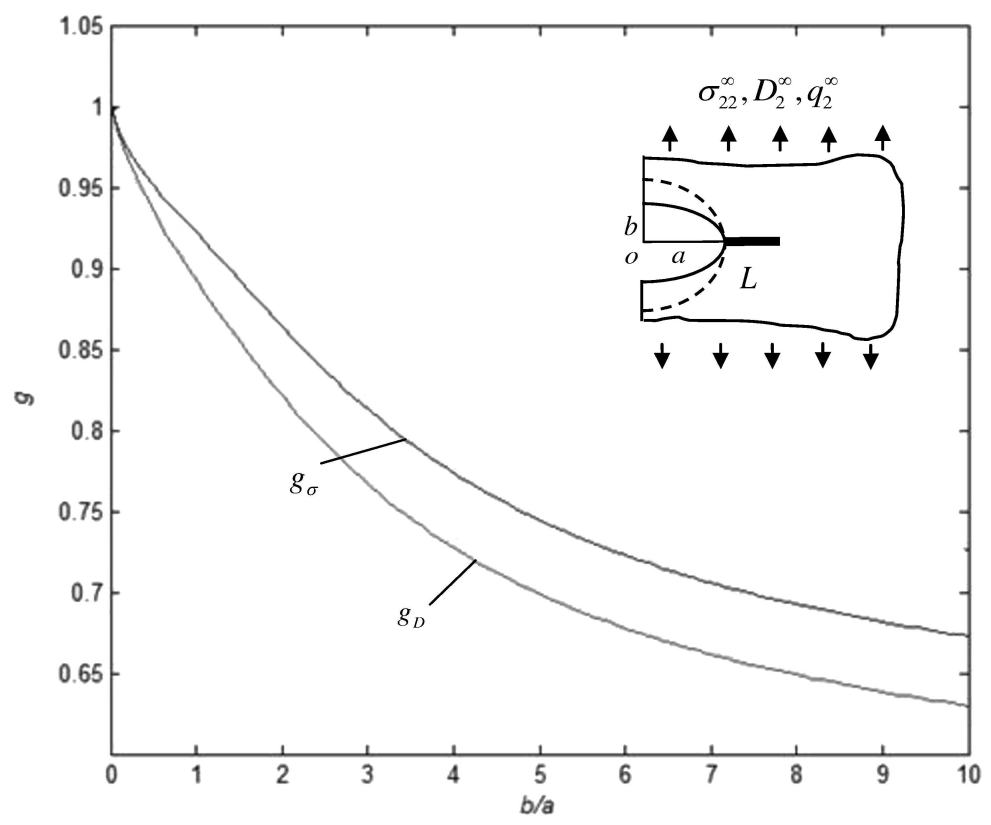

Fig. 8. Distribution of the equivalent fields intensity factors with the $b / a$ 
tip.

To discuss the influence of the heat flux on the FIF, we take $\sigma_{22}^{\infty}=0$ $\mathrm{MPa}, D_{2}^{\infty}=0 \mathrm{C} / \mathrm{m}^{-2}, L=0.01 \mathrm{~m} a=0.01 \mathrm{~m}$, and $b=0.5 a$. The distribution of the FIF with the heat flux is shown in Fig. 9. It can be seen, that as the heat flux increases, the stress intensity factor $k_{2}$ increases quickly, and $k_{1}$ increase slowly, but the electric displacement intensity factors $k_{D}$ are negative, when the heat flux perpendicular to the crack. That's because the thermal expansion at the surface of the crack are not equal, and the crack becomes a model II crack.

Let $L=b=0 \mathrm{~m}$, the cracked elliptical hole becomes a pure crack. The equivalent FIF can be expressed as:

$$
\mathbf{g}=1+\frac{2\left(\mathbf{B a}_{6}+\mathbf{d} a_{3}\right)}{a \varphi_{, 1}^{\infty}} .
$$

If there is no heat flux, $\left(\mathbf{B a}_{6}+\mathbf{d} a_{3}\right)=0$, and Eq. (71) equal to constant 1, which is the classical solution for the pure crack at the edge of a semi-infinite plane.

Ignoring the mechanical loading and Eq. (70) becomes:

$$
\mathbf{k}=\frac{2 \sqrt{\pi}}{\sqrt{\omega^{\prime \prime}(-1)}} \operatorname{Re}\left[\mathbf{B a}_{6}+\mathbf{d} a_{3}\right] .
$$

The FIF have the linear relationship with the heat flux, shown in Fig. 10. Compared with Fig. 9, the stress intensity factor $k_{2}$ for the pure crack $(a=0.02 \mathrm{~m})$ is a little bigger than the cracked elliptical hole $\left(a_{e}=0.02 \mathrm{~m}\right)$, which means the hole at the side of the crack can reduce the fields intensity factors.

\section{Conclution}

The generalized 2D problem of edged crack originating from a half elliptical hole in a half infinite thermo-electro-elastic solid is studied. Based on the complex variable method, combined with the conformal mapping approach, the complex potentials are derived for temperature fields and mechanical-electric fields, and then field intensity factors are presented. Numerical examples are also given to discuss the influences of the crack length and the heat flux on the electro-elastic fields. It is found, that:

1) The solution of the half plane is identical to the solution of the full plane; 


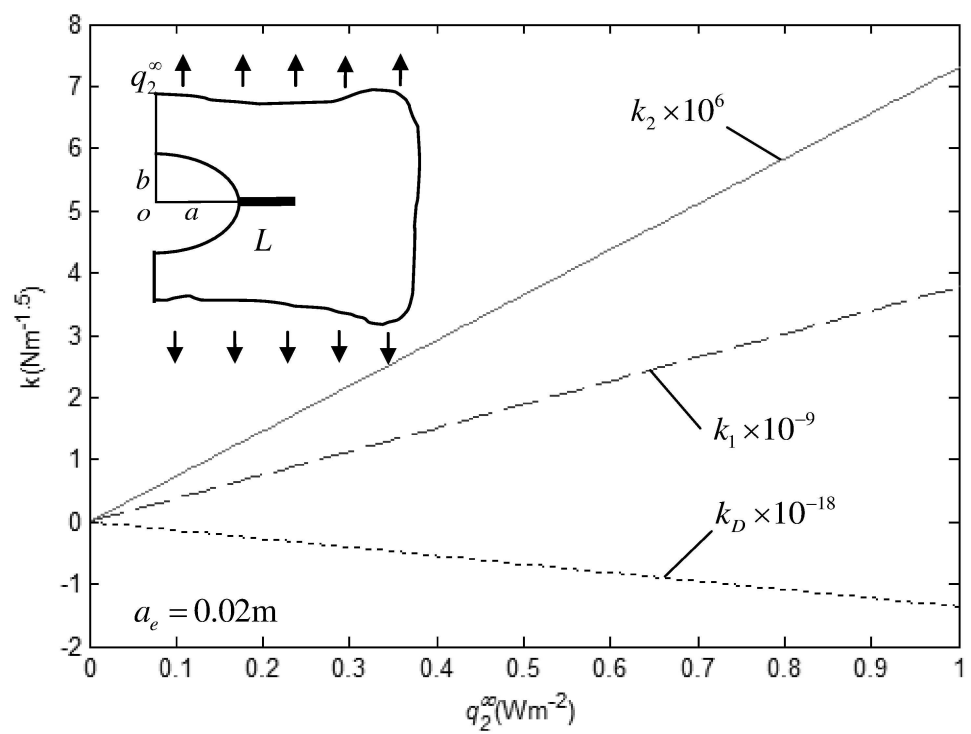

Fig. 9. Distribution of the field intensity factors with the heat flux (elliptical hole)

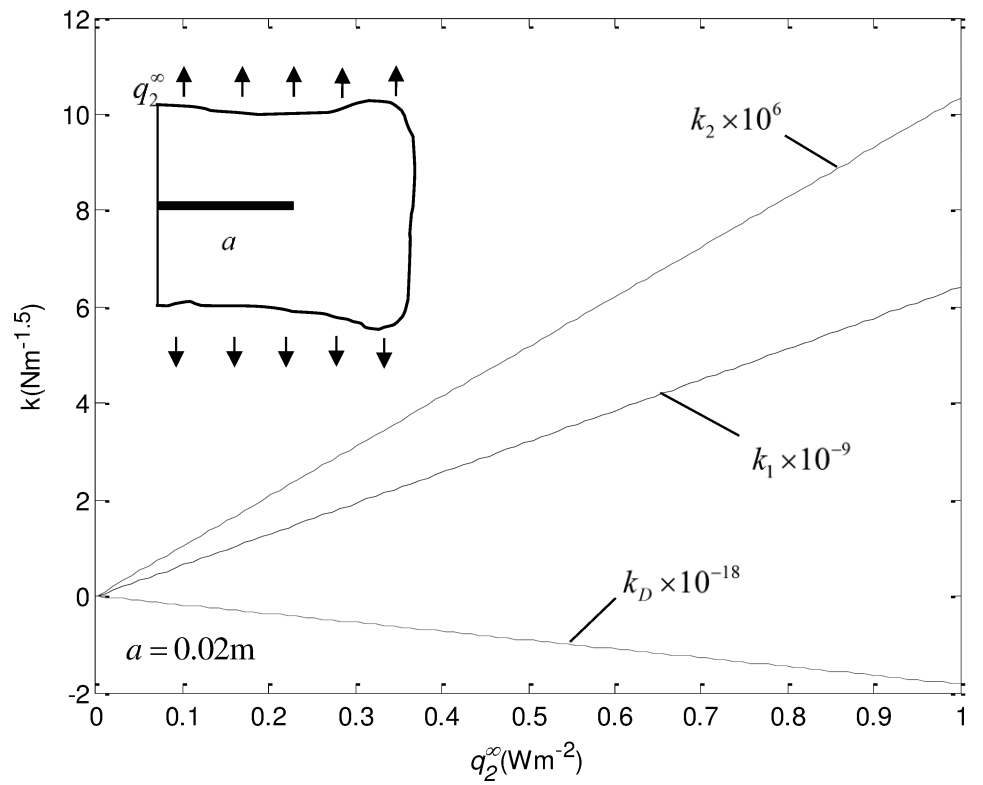

Fig. 10. Distribution of the field intensity factors with the heat flux (pure crack) 
2) The fields of heat flux, stresses, and electric displacement have the singularity of $r^{-0.5}$ at the crack tip, according to the boundary condition;

3) When the heat flux is perpendicular to the crack, the heat flux will have great effect on the stress intensity factors for the mode II cracks, while the influence on the stress intensity factors for the mode I cracks and the intensity factors of the electric displacements can be neglected;

4) The shape and the size of the hole have a significant effect on the field distribution at the crack tip, and the circular hole can effectively reduce the crack tip's singularity, in general.

\section{REFERENCES}

[1] Hetnarski, R. B., J. Ignaczak. The Mathematical Theory of Elasticity (Second edition), New York, Taylor \& Francis, 2011.

[2] Noda, N., R. B. Hetnarski, Y. Tanigawa. Thermal Stresses (Second edition), New York, Taylor \& Francis, 2003.

[3] Hetnarski, R. B., M. R. Eslami. Thermal Stresses - Advanced Theory and Applications, Springer, 2009.

[4] Tiersten, H. F. On the Nonlinear Equations of Thermo-electro-elasticity. Int. J. Eng. Sci., 9 (1971), 587-604.

[5] Chandrasekharaiah, D. S. A Generalized Linear Thermo-elasticity Theory for Piezoelectric Media. Acta. Mech., 71 (1988), 33-49.

[6] Gao, C. F., Y. T. Zhao, M. Z. Wang. An Exact and Explicit Treatment of an Elliptic Hole Problem in Thermo-piezo-electric Media. Int. J. Solids Struct., 39 (2002), 2665-2685.

[7] Hasebe, N., C. Bucher, R. Heuer. Heat Conduction and Thermal Stress induced by an Electric Current in an Infinite Thin Plate Containing an Elliptical Hole with an Edge Crack. Int. J. Solids Struct., 47 (2010), 138-147.

[8] GaO, C. F., M. Z. WANG. Collinear Permeable Cracks in Thermo-piezo-electric Materials. Mech. Mater., 33 (2001), 1-9.

[9] Wang, Y. J., C. F. GaO. The Mode III Cracks originating from the Edge of a Circular Hole in a Piezoelectric solid. Int. J. Solids Struct., 45 (2008), 4590-4599.

[10] Bowie, O. L. Analysis of an Infinite Plate containing Radial Cracks originating from the Boundary of an Internal Circular Hole. J. Math. Phys., 35 (1956), 60-71.

[11] Guo, J. H., Z. X. Lu, H. T. Han, Z. Y. YANG. Exact Solutions for Anti-plane Problem of Two Asymmetrical Edge Cracks emanating from an Elliptical Hole in a Piezoelectric Material. Int. J. Solids Struct., 46 (2009), 3799-3809.

[12] Guo, J. H., Z. X. Lu, H. T. Han, Z. Y. Yang. The Behaviour of Two Nonsymmetrical Permeable Cracks emanating from an Elliptical Hole in a Piezoelectric Solid. Eur. J. Mech. A-Solid., 29 (2010), 654-663. 
[13] Hasebe, N. Stress in Intensity Factors of Cracks initiating from a Rhombic Hole due to Uniform Heat Flux. Eng. Fract. Mech., 42 (1992), 331-337.

[14] Vinh, P. C., N. Hasebe, X. F. Wang. Interaction between a Cracked Hole and a Line Crack under Uniform Heat Flux. Int. J. Fracture, 131 (2005), 367-384.

[15] Wang, Y. J., C. F. Gao. Thermoelectroelastic Solution for Edge Cracks originating from an Elliptical Hole in a Piezoelectric Solid. J. Therm. Stresses, 35 (2012), 138-156.

[16] Lu, J. K. Complex Variable Method in the Elastic Plane. Wuhan, Wuhan University Press, 2005 (In Chinese).

[17] GaO, J., G. T. Liu. Stress Analysis of the Half Plane with a Half Elliptical Hole. Journal of Inner Mongolia Normal University, (Natural Science Edition), 40 (2011), 444-447 (In Chinese).

[18] Hasebe, N., S. Inohara. Stress Analysis of a Semi-infinite Plate with an Oblique Edge Crack. Arch. Appl. Mech., 49 (1980), 51-62.

[19] Chung, M. Y., T. C. T. Ting. Piezoelectric Solid with an Elliptic Inclusion or Hole. Int. J. Solids Struct., 33 (1996), 3343-3361.

[20] Shen, S., Z. B. Kunng. Interface Crack in Bi-piezothermoelastic Media and the Interaction with a Point Heat Source. Int. J. Solids Struct., 35 (1998), 3899-3915.

[21] Suo, Z. Singularities Interfaces and Cracks in Dissimilar Anisotropic Media. Proceedings of the Royal Society of London A, 427 (1990), 331-358.

[22] Muskhelishvili, N. I. Some Basic Problems of the Mathematical Theory of Elasticity, Groningen, Noordhoff, 1975.

[23] Ashida, F., J. S. Chol, N. Noda. Control of Elastic Displacement in Piezoelectric-based Intelligent Plate Subjected to Thermal Load. Int. J. Eng. Sci., 35 (1977), 851-868. 